\title{
Efficacy of Tenofovir Disoproxil Fumarate Therapy in Nucleoside-Analogue Naive Iranian Patients Treated for Chronic Hepatitis B
}

\author{
Fatemeh Bakhshizadeh ${ }^{1}$; Soheila Hekmat ${ }^{1, *}$; Maryam Keshvari ${ }^{2}$; Seyed Moayed Alavian ${ }^{3}$; \\ Ehsan Mostafavi ${ }^{4}$; Hossein Keivani ${ }^{5}$; Amin Doosti-Irani ${ }^{6}$; Fatemeh Motevalli ${ }^{1}$; Bita Behnava ${ }^{3}$ \\ ${ }_{1}^{1}$ Hepatitis and AIDS Department, Pasteur Institute of Iran, Tehran, IR Iran \\ ${ }_{2}^{2}$ Blood Transfusion Research Center, High Institute for Research and Education in Transfusion Medicine, Tehran, IR Iran \\ ${ }^{3}$ Middle East Liver Diseases Center (MELD), Tehran, IR Iran \\ 4 Epidemiology Department, Pasteur Institute of Iran, Tehran, IR Iran \\ 5 Virology Department, School of Medicine, Iran University of Medical Sciences, Tehran, IR Iran

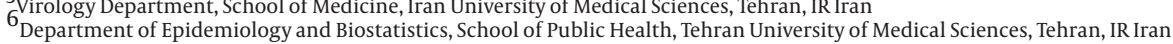 \\ *Corresponding Author: Soheila Hekmat, Hepatitis and AIDS Department, Pasteur institute of Iran, Tehran, IR Iran. Tel/Fax:+98-2166969291, E-mail: Sh_hekmat@yahoo.com
}

Received: December 1, 2014; Revised: January 28, 2015; Accepted: April 14, 2015

\begin{abstract}
Background: Tenofovir disoproxil fumarate (TDF) is a new effective treatment option for patients with chronic hepatitis B (CHB).
Objectives: To evaluate TDF efficacy in nucleos(t)ide analogues (NAs)-naive Iranian patients with CHB.

Patients and Methods: The NA-naive patients received TDF for at least six months. The primary endpoint was the proportion of patients achieving a complete virological response (CVR) during the treatment. Multivariate Cox regression analysis determined predictive factors independently associated with the time to CVR. The secondary endpoints were biochemical and serological responses, frequency of virological breakthrough, genotypic resistance development, safety and tolerability.

Results: In all, 93 patients (64.5\% hepatitis B e antigen [HBeAg]-negative) were eligible. Of these, 70 patients completed 24 months of treatment. The cumulative CVR rates in HBeAg-negative and HBeAg-positive patients were $87 \%$ versus $53 \%$ at 24 months, respectively. The multivariate Cox regression model showed only HBeAg positivity at baseline and a high baseline HBV DNA level were independent factors predicting a CVR. No patient achieved hepatitis B surface antigen (HBsAg) and HBeAg loss or seroconversion and no virologic breakthrough occurred. Anew amino acid substitution (rtD263E) was observed to develop in $60 \%$ of patients with viremia.

Conclusions: The cumulative CVR rates showed that patients with HBeAg-negative have better virologic respond than those with $\mathrm{HBeAg}$ positive during the same period. The rtD263E mutation might be associated with partial resistance to TDF.
\end{abstract}

Keywords: Chronic Hepatitis B; Tenofovir; nucleoside analogue

\section{Background}

Tenofovir disoproxil fumarate(TDF) is the prodrug of an acyclic nucleotide analogue with activity against hepatitis B virus (HBV). It is structurally closely related to adefovir and inhibits viral polymerase by direct binding and termination of DNA chain elongation (1).

TDF was approved in 2008 with dosage of $300 \mathrm{mg}$ once daily for the treatment of chronic hepatitis B (CHB). Its potent antiviral activity was confirmed by two randomized trials in mostly treatment-naive HBV monoinfected, hepatitis B e antigen (HBeAg)-positive and HBeAg-negative patients (2). TDF was also shown to durably suppress HBV replication and no drug resistant mutation has been reported within five years of continuous therapy $(3,4)$. An interesting finding about TDF is the relatively high rates of hepatitis B surface antigen (HBsAg) loss in HBeAgpositive patients $(2,3)$. However, there are overall fewer studies published with TDF than with other nucleos(t)ide analogs (NAs) and current data concerning TDF is mainly derived from multicenter clinical trials, which may not reflect the real efficacy and compliance for therapy (5). Moreover, TDF antiviral therapy has become a routine treatment for CHB patients in Iran in the recent years. Hence total evaluation of efficacy endpoints in TDF-treated CHB Iranian patients is warranted.

\section{Objectives}

The aim of this study was to evaluate and compare the efficacy of TDF therapy in HBeAg-positive and HBeAg-negative NA-naive patients infected chronically with HBV-D genotype (the most common genotype in Iran).

\section{Patients and Methods}

\subsection{Study Population}

This study included 93 NA-naive CHB patients, started to receive TDF between March 2011 and September 2013. Patients were followed up at Tehran Hepatitis Centre and Blood Transfusion Hepatitis Clinic (Tehran, IR Iran). Inclusion criteria were evidence of chronic HBV infection [i.e., HBsAg positive for more than six months, serum HBV DNA greater than $20000 \mathrm{IU}$ per $\mathrm{mL}$ (lower values of 2000 to

Copyright (C) 2015, Kowsar Corp. This is an open-access article distributed under the terms of the Creative Commons Attribution-NonCommercial 4.0 International License (http://creativecommons.org/licenses/by-nc/4.0/) which permits copy and redistribute the material just in noncommercial usages, provided the original work is properly cited. 
Bakhshizadeh F et al.

20000 IU per mL often occur with HBeAg-negative chronic hepatitis $B$ ), persistent or intermittent elevation in alanine transaminase (ALT) or aspartate transaminase (AST) levels and liver biopsy showing chronic hepatitis with moderate or severe necroinflammation] (6) and treatment with TDF (300 mg daily) for a minimum of six months. We excluded patients with coinfection by hepatitis $C$ virus, hepatitis D virus or HIV and those with previous or concurrent use of oral NAs before TDF treatment. Medical records were reviewed and information including patient demographics (age, gender), HBV markers (HBeAg, antibody against HBe [anti-HBe], HBsAg, antibody against HBs [anti-HBs] and HBV DNA), hepatic panel (aspartate aminotransferase [AST], alanine aminotransferase [ALT], total bilirubin, alpha-fetoprotein and serum creatinine) and liver histology were recorded. This study was approved by the Iranian Hepatitis Network ethics committee and an informed written consent was obtained from all patients.

\subsection{Clinical Endpoints}

The primary endpoint of this study was the proportion of patients achieving a complete virological response (CVR), defined as undetectable serum HBV DNA level (< $6 \mathrm{IU} / \mathrm{mL}$ ) during the TDF treatment. Univariate and multivariate Cox regression analyses were performed to find independent factors that influence achieving CVR. The secondary endpoints were ALT normalization (upper limit of the normal range: $34 \mathrm{U} / \mathrm{L}$ [female patients] and 40 $\mathrm{U} / \mathrm{L}$ [male patients]), HBeAg and HBsAg loss or seroconversion, determination of the frequency and causes of virological breakthrough, defined as an increase of serum HBV DNA level by at least 1 log from the nadir during the TDF treatment (7), genotypic resistance development and drug safety and tolerability. These primary and secondary endpoints were compared between HBeAg-positive and HBeAg-negative patients.

\subsection{Clinical and Laboratory Assessments}

Patients were routinely assessed every 6 - 12 months after starting the TDF treatment. Serum HBV DNA was quantitated by Cobas TaqMan, Roche Diagnostics, with a lower detection limit of $6 \mathrm{IU} / \mathrm{mL}$, and other viral markers (HBeAg, anti-HBe, HBsAg and anti-HBs levels) were measured using the ELISA method (8). In addition, serum HBsAg was quantitated using HBsAg II quant assay (Roche Diagnostics, Germany).

Direct sequencing was conducted to assess genotypic resistance development in the HBV polymerase-reverse transcriptase (RT) by an automated sequencer at the SEQLAB-Gottingen GmbH, Germany. The obtained sequences were investigated for all classical and non-classical mutations described to be associated with resistance against NAs in previous studies (9). In addition, the new amino acid substitutions were identified by comparing HBV RT sequences with the genotype-matched consensus sequence from Iranian isolates obtained from GenBank.

\subsection{Statistical Analyses}

Statistical analyses were performed using Stata/SE 12.0 (Stata Corp. 2011. Stata Statistical Software: Release 12. College Station, TX: Stata Corp LP.). Continuous variables are presented as means \pm standard deviations or medians (ranges) and all categorical variables as frequencies (percentages). One-way ANOVA test was used for comparing quantitative variables and chi-square test for comparisons involving categorical variables. The cumulative rates of virologic suppression (HBV DNA $<6 \mathrm{IU} / \mathrm{mL}$ ) were calculated using the Kaplan-Meier method and time to event subgroup comparisons were performed using LogRank test. Cox proportional-hazard model was used to identify predictive factors independently associated with the time to a CVR. The results of the model are presented as a hazard ratio (HR) with the $95 \%$ confidence interval (CI). A Pearson's correlation coefficient was used to assess the correlation between HBV DNA and HBsAg. P $<0.05$ was considered statistically significant.

\section{Results}

\subsection{Baseline Characteristics of Patients}

Ninety-three patients were included in this study. Of these, 84 (90\%) patients received TDF for at least 12 months and 70 (75\%) patients completed 24 months of therapy. The mean duration of treatment with TDF was 21.10 (ranged 6 to 36) months and the durations were similar for both HBeAg-negative and HBeAg-positive patients. The baseline clinical, demographic and laboratory characteristics of 93 patients are summarized in Table 1 . The number of patients with HBeAg-negative and HBeAg-positive CHB were 60 (64.5\%) and 33 (35.5\%), respectively. The mean baseline HBV DNA levels were significantly higher in patients with HBeAg-positive than those with HBeAg-negative (8.67 \pm 8.84 versus $8.07 \pm 8.54 \log _{10} \mathrm{IU} / \mathrm{mL}, \mathrm{P}=0.002$, respectively). Pretreatment ALT level was higher in patients with HBeAgpositive, but the difference was not statistically significant (mean $77 \pm 43$ versus $70 \pm 60 \mathrm{IU} / \mathrm{L}, \mathrm{P}=0.605$ ).

\subsection{Virologic Response}

To assess the efficacy of TDF treatment, CVR rates were calculated for all time points of TDF treatment by KaplanMeier analysis. The overall proportion of CVR during the follow-up was $67 \%$ (62/93) in all patients. These values were $80 \%$ (48/60) and 42\% (14/33) in patients with HBeAgnegative and HBeAg-positive, respectively, which the difference was statistically significant $(P=0.000)$. The cumulative CVR rates in HBeAg-negative and HBeAg-positive patients were $53 \%$ versus $18 \%$ at six months, 74 versus $38 \%$ at 12 months and $87 \%$ versus $53 \%$ at 24 months, respectively (Figure 1 ; log-rank test, $\mathrm{P}=0.000$ ).

No virologic breakthrough occurred during the followup period. Univariate Cox regression analyses of all patients revealed that age ( $\mathrm{HR}, 0.97 ; 95 \% \mathrm{CI}, 0.93$ to $0.99 ; \mathrm{P}$ $=0.035$ ), HBeAg status at baseline (HR, 0.42; 95\% CI, 0.24 
Bakhshizadeh F et al.

to $0.73 ; \mathrm{P}=0.002$ ), a high baseline ALT level (above the upper limit of the normal range [ULN]) (HR, 0.98; 95\% CI, 0.96 to $0.99 ; \mathrm{P}=0.044)$ and a high baseline HBV DNA level $(>2 \times 106 \mathrm{IU} / \mathrm{mL})(\mathrm{HR}, 1 ; 95 \% \mathrm{CI}, 1$ to $1 ; \mathrm{P}=0.001)$ were associated with CVR achievement (Table 2 ). In multivariate Cox proportional-hazard analysis, only HBeAg positivity at baseline (HR, 0.42; 95\% CI, 0.22 to $0.81 ; \mathrm{P}=0.010$ ) and a high baseline HBV DNA level (HR, 1; 95\% CI, 1 to $1 ; \mathrm{P}=0.043$ ) were independent factors predicting a CVR (Table 3 ).

\subsection{Biochemical Response}

Overall, 72\% (67/93) of patients achieved ALT normalization during the follow-up. These values were 75\% (45/60) and $67 \%(22 / 33)$ in patients with HBeAg-negative and HBeAg-positive, respectively, which the difference was not statistically significant $(P=0.181)$. The cumulative rates of ALT normalization in HBeAg-negative and HBeAgpositive patients who had high baseline ALT levels were $65 \%$ versus $57 \%$ at six months, $74 \%$ versus $74 \%$ at 12 months and $74 \%$ versus $78 \%$ at 24 months, respectively (Figure 2 ; log-rank test, $\mathrm{P}=0.935$ ).

\subsection{Serological Response}

No patient achieved HBeAg and HBsAg loss or seroconversion during the follow-up. The median HBsAg level at baseline was 3.83 (range, 2.11 - 5.28) $\log \mathrm{IU} / \mathrm{mL}$. This value was significantly higher in patients with HBeAg-positive than those with HBeAg-negative (4.25 [range, 3.12 - 5.28] versus 3.53 [range, $3.05-4.34$ ] $\log \mathrm{IU} / \mathrm{mL}, \mathrm{P}=0.002$, respectively). After a mean duration of 15 months (range, 12 - 24) of TDF treatment, the median rate of HBsAg reduction was 2.98 (range, -4.00 - 5.22) $\log \mathrm{IU} / \mathrm{mL}$. These values were 2.64 (range, -4.00 - 4.87) and 3.57 (range, -2.70 - 5.22) log IU/ $\mathrm{mL}$ in patients with HBeAg-negative and HBeAg-positive, respectively, which the difference was statistically significant $(\mathrm{P}=0.049)$. There was a correlation between baseline HBsAg levels with both baseline HBV DNA levels ( $\mathrm{r}=$ $0.51, \mathrm{P}=0.000)$ and the rate of HBsAg reduction at a mean duration of 15 months $(r=0.44, \mathrm{P}=0.019)$. The rate of HB$\mathrm{sAg}$ reduction showed no correlation with both baseline
HBV DNA levels $(r=0.17, \mathrm{P}=0.379)$ and the rate of $\mathrm{HBV}$ DNA reduction over the same time $(\mathrm{r}=0.34, \mathrm{P}=0.086)$. This correlation was not observed in both HBeAg-positive and HBeAg-negative patients $(r=0.24, P=0.476$ versus $r=$ $0.31, \mathrm{P}=0.262$, respectively).

\subsection{Resistance Surveillance}

A genotypic analysis of 50 patients with viremia (HBV DNA $\geq 6 \mathrm{IU} / \mathrm{mL}$ ) at their last time point on treatment (the mean duration of 14 months) was conducted. No patient harbored previously described amino acid substitutions, including substitutions that could be associated with reduced TDF susceptibility (rtA181V/T, rtN236T or rtA194T). Moreover, a comparison between baseline and post-baseline samples from these patients showed that the presence of rtL91I, rtN238H/T/S, rtC256G, rtN53S/T and rtY54N mutations reduced significantly after the initiation of TDF treatment $(\mathrm{P}<0.05)$ (Table 4$)$. These mutations associated with resistance against lamivudine (LAM) or adefovir dipivoxil (ADV) at previous reports. In contrast, a new amino acid substitution (rtD263E) was found exclusively in $60 \%$ (30/50) of patients treated with TDF, while this mutation was only observed in $12 \%$ (6/50) of patients prior to initiation of TDF therapy.

\subsection{Safety}

TDF treatment was well tolerated and there were no serious adverse events reported in the present study. The serum creatinine level changed during the TDF therapy, from a mean of $0.98 \pm 0.26$ (range, 0.10 to 1.79 ) $\mathrm{mg} / \mathrm{dL}$ at baseline to $1.02 \pm 0.17$ (range, 0.70 to 1.38 ) $\mathrm{mg} / \mathrm{dL}$ at the end of follow-up. Overall, three patients experienced a $\geq$ $0.5 \mathrm{mg} / \mathrm{dL}$ increase in creatinine. However, these patients remained on study through 24 months without modification of TDF treatment. No ALT flares $(>5 \times$ upper limit of normal) were observed during the follow-up. A male patient developed compensated cirrhosis during the follow-up. This patient remained in remission and responded well to TDF therapy. HCC occurrence was not detected in any patient during the TDF treatment.

\begin{tabular}{|c|c|c|c|c|}
\hline Characteristic & Overall $(\mathbf{n}=93)$ & HBeAg-Positive $(\mathrm{n}=33)$ & HBeAg-Negative $(\mathbf{n}=60)$ & Pvalue \\
\hline Male $^{\mathrm{b}}$ & $72(77)$ & $26(79)$ & $46(77)$ & 1.000 \\
\hline $\operatorname{Age}^{c}, y$ & $38 \pm 12$ & $31 \pm 10$ & $42 \pm 11$ & 0.000 \\
\hline ALT level ${ }^{\mathrm{C}}$, IU/L & $73 \pm 54$ & $77 \pm 43$ & $70 \pm 60$ & 0.605 \\
\hline ALT level above ULN ${ }^{b}$ & $67(72)$ & $30(91)$ & $37(62)$ & 0.001 \\
\hline HBV DNA level ${ }^{\mathrm{C}}, \log _{10} \mathrm{IU} / \mathrm{mL}$ & $8.38 \pm 8.71$ & $8.67 \pm 8.84$ & $8.07 \pm 8.54$ & 0.002 \\
\hline AST level ${ }^{\mathrm{C}}$, IU/L & $53 \pm 53$ & $57 \pm 52$ & $51 \pm 54$ & 0.609 \\
\hline Total bilirubin level ${ }^{\mathrm{c}}$, mg/dL & $1.06 \pm 0.77$ & $1.16 \pm 0.86$ & $1.01 \pm 0.72$ & 0.379 \\
\hline Alpha-fetoprotein level ${ }^{C}, \mathrm{mg} / \mathrm{dL}$ & $6.87 \pm 13.41$ & $10.36 \pm 20.32$ & $5.00 \pm 7.42$ & 0.200 \\
\hline Liver cirrhosis ${ }^{b}$ & $8(9)$ & $4(12)$ & $4(7)$ & 0.448 \\
\hline Treatment duration $^{\mathrm{d}}$, mo & $21.10(6-36)$ & $18.18(6-36)$ & $22.70(6-36)$ & \\
\hline
\end{tabular}


Bakhshizadeh F et al.

\begin{tabular}{|c|c|c|c|c|}
\hline \multirow{2}{*}{ Factor } & \multirow{2}{*}{ Hazard Ratio } & \multicolumn{2}{|c|}{ 95\% Confidence Interval } & \multirow{2}{*}{ Pvalue } \\
\hline & & Lower & Upper & \\
\hline Gender $^{\mathrm{C}}$ & 0.92 & 0.40 & 2.15 & 0.855 \\
\hline Age & 0.97 & 0.93 & 0.99 & 0.035 \\
\hline Cirrhosis & 0.33 & 0.04 & 2.40 & 0.271 \\
\hline HBeAg status ${ }^{d}$ & 0.42 & 0.24 & 0.73 & 0.002 \\
\hline High baseline ALT level ${ }^{e}$ & 0.98 & 0.96 & 0.99 & 0.044 \\
\hline High baseline HBVDNA, $>2 \times 10^{6} \mathrm{IU} / \mathrm{mL}$ & 1.00 & 1.00 & 1.00 & 0.001 \\
\hline \multicolumn{5}{|c|}{$\begin{array}{l}\text { a Abbreviations: ALT, Alanine aminotransferase; CVR, Complete virological response. } \\
\text { b CVR is HBV DNA }<6 \mathrm{IU} / \mathrm{mL} \text {. } \\
\text { c Data are presented for males. } \\
\text { d The HBeAg positive was the indicator variable for HBeAg status in the analysis. }\end{array}$} \\
\hline
\end{tabular}

\begin{tabular}{|c|c|c|c|c|}
\hline \multirow{2}{*}{ Factor } & \multirow{2}{*}{ Hazard Ratio } & \multicolumn{2}{|c|}{ 95\% Confidence Interval } & \multirow{2}{*}{ P value } \\
\hline & & Lower & Upper & \\
\hline Age & 1.01 & 0.99 & 1.04 & 0.317 \\
\hline HBeAg status ${ }^{C}$ & 0.42 & 0.22 & 0.81 & 0.010 \\
\hline High baseline ALT level & 1.00 & 0.10 & 1.00 & 0.727 \\
\hline High baseline HBVDNA, > $2 \times 106 \mathrm{IU} / \mathrm{mL}$ & 1.00 & 1.00 & 1.00 & 0.043 \\
\hline
\end{tabular}

${ }^{\mathrm{a}}$ Abbreviations: ALT, Alanine aminotransferase; CVR, Complete virological response.

b CVR is HBV DNA $<6 \mathrm{IU} / \mathrm{mL}$.

c The HBeAg positive was the indicator variable for HBeAg status in the analysis.

\begin{tabular}{lcccc}
\hline \multicolumn{4}{l}{ Table 4. Genotypic Analysis Results for Patients With Viremia During the TDF Therapy a,b } & \\
\hline \multicolumn{1}{l}{ Type } & Baseline & Last Time Point on Treatment & Pvalue & Associated Resistance [20] \\
\hline Patients withN53K/D/H & $9(18)$ & $2(4)$ & 0.036 & LAM \\
Patients with Y54N/H & $16(32)$ & $4(8)$ & 0.002 & ADV \\
Patients with L91I & $5(10)$ & $0(0)$ & 0.020 & LAM \\
Patients with N238H/T & $5(10)$ & $0(0)$ & 0.020 & ADV \\
Patients with C256G/S & $11(22)$ & $3(6)$ & 0.030 & LAM \\
\hline
\end{tabular}

a Abbreviations: ADV, Adefovir Dipivoxil; LAM, Lamivudine.

b Data are presented as No. (\%).

Figure 1. Cumulative Complete Virological Response (CVR) Rates in HBeAg Positive and Negative Patients

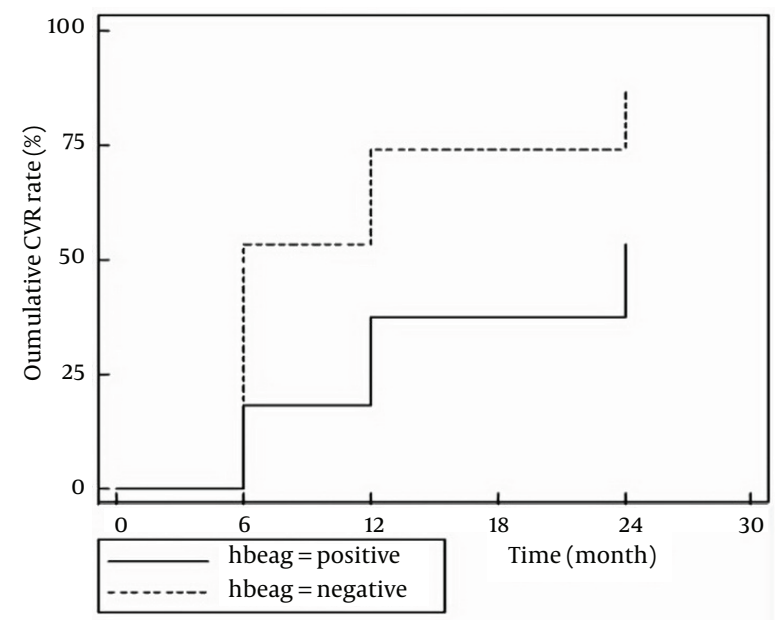

Analysis was performed by the Kaplan-Meier method; log-rank test, $\mathrm{P}=$ 0.00 .
Figure 2. Cumulative Alanine Aminotransferase (ALT) Normalization Rates in HBeAg Positive and Negative Patients

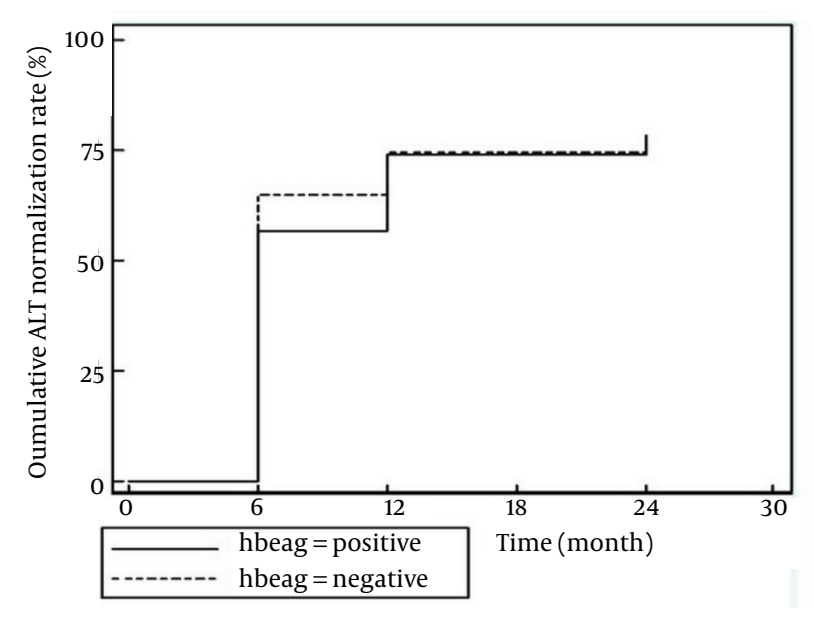

Analysis was performed by the Kaplan-Meier method; log-rank test, $\mathrm{P}=$ 0.9 . 
Bakhshizadeh F et al.

\section{Discussion}

In the present study, we followed the efficacy of TDF therapy in Iranian NA-naive patients in 24 months. Consistent with previous studies, TDF produced effective viral suppression in both HBeAg-negative and HBeAg-positive patients $(2,3,10)$. However, the CVR rates of HBeAg-negative patients were significantly higher than those with HBeAg-positive. This is probably due to higher pretreatment HBV DNA levels in HBeAg-positive patients. The results of the multivariate analysis confirmed that only a high baseline HBV DNA level and HBeAg status, which is associated with the baseline viral load, were independent predictors of the time achieving a CVR. In previous studies, baseline HBV DNA levels and HBeAg status were appeared to predict HBV DNA clearance in clinical practice, which are consistent with the results obtained in our study $(11,12)$.

Biochemical response rate was also different between HBeAg-negative and HBeAg-positive patients. However, the difference was not significant. No HBeAg loss and seroconversion were observed in HBeAg-positive patients, which is different from that reported in previous studies $(2,13)$. For example, in a randomized trial, comparing Tenofovir and adefovir in $266 \mathrm{HBeAg}$-positive patients, the HBeAg seroconversion rates at 1 and 2 years of TDF therapy were 21 and 26\%, respectively (2). In HBeAg-positive patients, on-treatment HBV DNA levels at week 24 appeared to be the most robust predictor of response, including HBeAg seroconversion $(14,15)$. This may explain lack of HBeAg seroconversion in our population, so that in the mentioned study, $49 \%$ of HBeAg-positive patients achieved an undetectable HBV DNA level at the end of 24 weeks of follow-up, while this value was only $18 \%$ in our patients. Moreover, this result may be obtained due to limited number of HBeAg-positive patients (33/93) in our study. We did not identify any patient with HBsAg loss during the follow-up. However, the results showed an overall strong decline in median HBsAg levels occurred from baseline to mean duration of 15 months. The decline of HBsAg during NA therapy may reflect a better degree of host immune control against the virus and it has been shown to have a good correlation with reduction in cccDNA during antiviral therapy $(16,17)$. The HBsAg levels at baseline and the rate of HBsAg reduction during the observation period were significantly higher in patients with HBeAg-positive than those with HBeAg-negative. This aspect of TDF efficacy has been confirmed in previous studies in patients receiving NAs, indicating that decline of HBsAg appears more apparent in HBeAg-positive patients than HBeAg-negative ones $(3,18,19)$. In addition, the rate of HBsAg reduction in $\mathrm{HBeAg}$-negative patients were significantly lower than HBeAg-positive ones, but there was a strong decline in HBsAg level. This is in contrast to findings of a previous study showing that long-term therapy with TDF and entecavir do not result a clinically significant HBsAg decline in HBeAg negative patients (20). Our study found that the rate of serum HBV DNA and HBsAg reduction were not correlated in the same period, similar to studies conducted so far $(18,21)$. In fact, the amount of decline in HBsAg level during the observation period was not associated with higher rates of complete HBV DNA suppression or HBeAg seroconversion.

In genotypic analysis of samples from patients with viremia at their last time point on-treatment, classical mutations which could be associated with reduced TDF susceptibility were not detected in any patient. In addition, two mutations of rtL91I and rtN238H/T/S presented in $10 \%$ of baseline sequences from patients, were not detected in these patients after the treatment with TDF. The other three non-classical mutations, including rtC256G, rtN53S/T and rtY54N observed in $6 \%, 4 \%$ and $8 \%$ of patients with viremia, respectively, were detected significantly lower than pretreatment time. These substitutions potentially associated with ADV or LAM resistance or replication compensation (9). In fact, antiviral pressure exerted by TDF-based therapy suppressed mutant and wild-type virus enrichment. One can argue that wild-type HBV had a fitness advantage than the ADV-and/or LAM-resistant mutants even in the presence of TDF, which would lead to earlier disappearance of these strains, compared to wildtype strains. These data suggest the necessity of further evaluation in in vitro and in vivo studies.

We also identified one new amino acid substitution as rtD263E in the HBV RT. The rtD263E is located after an E domain at RT-area and in the C-term part of the palm domain with $65.5 \%$ conserved amino acids that do not overlap with HBsAg region. Although this change was not associated with virological breakthrough, it was observed to develop in $60 \%$ of patients with viremia on TDF therapy. The emergence of this substitution in most patients with viremia under the treatment with TDF may be the reason for incomplete response to TDF in these patients.

TDF therapy showed a suitable safety and tolerability profile in patients, which is congruent with previous studies $(2,3,10)$. In conclusion, TDF is an effective and well-tolerated antiviral agent for the treatment of NA-naive patients with $\mathrm{CHB}$. HBeAg-negative patients have better virological responses than HBeAg-positive ones in the same period. Although TDF treatment showed no HBeAg and HBsAg loss or seroconversion in patients, it strongly decreased the HBsAg level in both HBeAg-negative and exclusively HBeAg-positive patients. This data suggests that HBsAg loss would occur in a shorter period in TDF therapy. In the present study, a new amino acid substitution (rtD263E) was observed to develop in $60 \%$ of patients with viremia on TDF therapy. Further studies are warranted to determine whether rtD263E mutation is associated with partial resistance to TDF, an anti-HBV molecule with the highest resistance barrier.

\section{Authors' Contributions}

Study concept and design: Soheila Hekmat. Acquisition 
of data: Fatemeh Bakhshizadeh, Seyed Moayed Alavian, Hossein Keivani, Maryam Keshvari and Bita Behnava. Drafting of the manuscript: Fatemeh Bakhshizadeh. Critical revision of the manuscript for important intellectual content: Soheila Hekmat and Maryam Keshvari. Statistical analysis: Ehsan Mostafavi and Amin Doosti. Administrative and technical supports: Fatemeh Motevalli.

\section{Funding/Support}

This investigation was supported by Pasteur Institute of Iran, Tehran, Iran.

\section{References}

1. Heijtink RA, Kruining J, de Wilde GA, Balzarini J, de Clercq E, Schalm SW.Inhibitory effects of acyclic nucleoside phosphonates on human hepatitis B virus and duck hepatitis B virus infections in tissue culture. Antimicrob Agents Chemother. 1994;38(9):2180-2.

2. Marcellin P, Heathcote EJ, Buti M, Gane E, de Man RA, Krastev Z et al. Tenofovir disoproxil fumarate versus adefovir dipivoxil for chronic hepatitis B. N Engl J Med. 2008;359(23):2442-55.

3. Heathcote EJ, Marcellin P, Buti M, Gane E, De Man RA, Krastev Z, et al. Three-year efficacy and safety of tenofovir disoproxil fumarate treatment for chronic hepatitis B. Gastroenterology. 2011; 140(1):132-43.

4. Marcellin P, Gane E, Buti M, Afdhal N, Sievert W, Jacobson IM, et al. Regression of cirrhosis during treatment with tenofovir disoproxil fumarate for chronic hepatitis B: a 5-year open-label follow-up study. Lancet. 2013;381(9865):468-75.

5. Ward JW, Lok AS, Thomas DL, El-Serag HB, Kim WR. Report on a single-topic conference on "Chronic viral hepatitis--strategies to improve effectiveness of screening and treatment". Hepatology. 2012;55(1):307-15.

6. Huntzinger A. AASLD Updates Chronic Hepatitis B Recommendations. Am Fam Physician. 2009;79(4):338-43.

7. Lok AS, McMahon BJ. Chronic hepatitis B. Hepatology. 2007; 45(2):507-39.

8. Yuen MF, Yuan HJ, Wong DK, Yuen JC, Wong WM, Chan AO, et al. Prognostic determinants for chronic hepatitis B in Asians: therapeutic implications. Gut. 2005;54(11):1610-4.

9. Liu BM, Li T, Xu J, Li XG, Dong JP, Yan P, et al. Characterization of potential antiviral resistance mutations in hepatitis $B$ virus reverse transcriptase sequences in treatment-naive Chinese patients. Antiviral Res. 2010;85(3):512-9.
10. Gordon SC, Krastev Z, Horban A, Petersen J, Sperl J, Dinh P, et al. Efficacy of tenofovir disoproxil fumarate at 240 weeks in patients with chronic hepatitis B with high baseline viral load. Hepatology. 2013;58(2):505-13.

11. Ridruejo E. Treatment of chronic hepatitis B in clinical practice with entecavir or tenofovir. World J Gastroenterol. 2014; 20(23):7169-80.

12. Baran B, Soyer OM, Ormeci AC, Gokturk S, Evirgen S, Bozbey HU, et al. Efficacy of tenofovir in patients with Lamivudine failure is not different from that in nucleoside/nucleotide analogue-naive patients with chronic hepatitis B. Antimicrob Agents Chemother 2013;57(4):1790-6.

13. Lin B, Ha NB, Liu A, Trinh HN, Nguyen HA, Nguyen KK, et al. Low incidence of hepatitis $B$ e antigen seroconversion in patients treated with oral nucleos(t)ides in routine practice. J Gastroenterol Hepatol. 2013;28(5):855-60.

14. Kau A, Vermehren J, Sarrazin C. Treatment predictors of a sustained virologic response in hepatitis B and C. J Hepatol. 2008;49(4):634-51.

15. Zeuzem S, Gane E, Liaw YF, Lim SG, DiBisceglie A, Buti M, et al Baseline characteristics and early on-treatment response predict the outcomes of 2 years of telbivudine treatment of chronic hepatitis B. J Hepatol. 2009;51(1):11-20.

16. Werle-Lapostolle B, Bowden S, Locarnini S, Wursthorn K, Petersen $J$, Lau G, et al. Persistence of cccDNA during the natural history of chronic hepatitis $B$ and decline during adefovir dipivoxil therapy. Gastroenterology. 2004;126(7):1750-8.

17. Chan HL, Wong VW, Tse AM, Tse CH, Chim AM, Chan HY, et al. Serum hepatitis B surface antigen quantitation can reflect hepatitis B virus in the liver and predict treatment response. Clin Gastroenterol Hepatol. 2007;5(12):1462-8.

18. Fung J, Lai CL, Young J, Wong DK, Yuen J, Seto WK, et al. Quantitative hepatitis B surface antigen levels in patients with chronic hepatitis B after 2 years of entecavir treatment. Am J Gastroenterol. 2011;106(10):1766-73.

19. Lee JM, Ahn SH, Kim HS, Park H, Chang HY, Kim do Y, et al. Quantitative hepatitis B surface antigen and hepatitis B e antigen titers in prediction of treatment response to entecavir. Hepatology. 2011;53(5):1486-93.

20. Zoutendijk R, Hansen BE, van Vuuren AJ, Boucher CA, Janssen HL. Serum HBsAg decline during long-term potent nucleos(t)ide analogue therapy for chronic hepatitis B and prediction of HBsAg loss. J Infect Dis. 2011;204(3):415-8.

21. Wursthorn K, Jung M, Riva A, Goodman ZD, Lopez P, Bao W, et al. Kinetics of hepatitis B surface antigen decline during 3 years of telbivudine treatment in hepatitis B e antigen-positive patients. Hepatology. 2010;52(5):1611-20. 